\title{
INVESTIGACIONES
}

\section{Las estrategias docentes al servicio del desarrollo del aprendizaje autorregulado}

\author{
Teaching strategies for self-regulated learning \\ As estratégias do ensino para o desenvolvimento \\ da autorregulação da aprendizagem
}

\section{Florencia Daura}

CIAFIC (Centro de Investigación en Antropología Filosófica y Cultural), CONICET. Buenos Aires. Argentina, fdaura@austral.edu.ar, (0054) (11) 476-0913

\begin{abstract}
RESUMEN
El artículo presenta un marco teórico en el que se considera la didáctica como saber de carácter teórico práctico, y describe los resultados preliminares de una investigación en curso, referidos a datos recogidos en observaciones de clase realizadas en la carrera de medicina, trabajo para el cual se utilizó una guía que podría ser aplicada en otros contextos universitarios.
\end{abstract}

Palabras clave: didáctica en la universidad, estrategias didácticas, aprendizaje autorregulado.

\begin{abstract}
Within a theoretical framework that regards teaching methodology as a theoretical and practical knowledge, this paper presents the preliminary results of an ongoing inquiry based on classroom observation data gathered at a medicine school. It is suggested that the working guidelines used might be applicable in other university contexts.
\end{abstract}

Key words: university teaching methodology, teaching strategies, self-regulated learning.

\section{RESUMO}

Apresenta um marco teórico no qual se considera a Didática como saber de caráter teórico e prático e descreve resultados preliminares de uma investigação em curso, obtidos por meio de coleta de dados observados em aulas do curso de Medicina. Utilizou-se um guia que poderia ser aplicado em outros contextos universitários.

Palavras chave: didática na universidade, estratégias didáticas, autorregulação da aprendizagem. 


\section{INTRODUCCIÓN}

El docente universitario es uno de los responsables de estimular en los estudiantes los conocimientos propios de la disciplina que enseña y las competencias que son necesarias para la adaptación en el mundo laboral, entre las que se destaca especialmente el aprendizaje autónomo o autorregulado.

El reconocimiento de la didáctica como una disciplina de carácter teórico y práctico, facilitaría la utilización de procedimientos, técnicas y recursos en base a los objetivos, a los contenidos, al contexto y a los destinatarios del proceso mencionado, cuestión que hace alusión al desenvolvimiento de una capacidad estratégica para enseñar, que puede ser adquirida gracias a una actitud de predisposición y de evaluación del propio desempeño como docente.

De acuerdo con estos lineamientos, el objetivo del presente trabajo es profundizar en el estudio de varios conceptos propios de la didáctica para repensarlos a la luz de los datos obtenidos a través de numerosas observaciones llevadas a cabo en el aula universitaria, considerando cuáles de entre las estrategias utilizadas podrían facilitar el desarrollo del aprendizaje autónomo en el estudiante.

\section{LA DIDÁCTICA, UNA DISCIPLINA PEDAGÓGICA DE CARÁCTER APLICATIVO}

En el presente trabajo, la didáctica es concebida como una disciplina que puede llegar a desarrollar modelos de acción y de reflexión que respondan a cuatro interrogantes básicos: ¿Para qué formar a los estudiantes y al profesorado? ¿Quiénes son nuestros estudiantes y cómo aprenden? ¿Qué se ha de enseñar y qué supone actualizar el saber? y ¿Cómo y con qué medios se puede realizar la enseñanza?, que sirvan para comprender la tarea desarrollada por los docentes y los alumnos en el proceso de enseñanza-aprendizaje (Medina Rivilla y Salvador Mata, 2005).

Esta visión exige que el profesor evite la adopción de una postura pasiva, aprovechando con creatividad y amplitud los recursos propios de la disciplina y los factores que en ella confluyen. Para ello, tendrá que aplicar una visión estratégica con la que pueda regular su acción y así promover en el estudiante el desarrollo de algunas variables que entran en juego en el aprendizaje autónomo, tales como las cognitivas, motivacionales, afectivas y contextuales (Pintrich y De Groot, 1990).

\subsection{ESTRATEGIAS DE APRENDIZAJE Y DE ENSEÑANZA}

Gracias a la interacción que se produce en el proceso de aprendizaje, docentes y discentes se desenvuelven como enseñantes o aprendices, roles que pueden intercambiar en base al momento del proceso en el que se encuentren y el estilo personal que cada uno posea. De allí que las estrategias de aprendizaje y las de enseñanza pueden ser utilizadas por unos u otros.

Las estrategias de aprendizaje son procedimientos que el aprendiz utiliza en forma consciente, regulada, intencional y flexible para enfrentarse a situaciones problemáticas y para aprender en forma significativa alcanzando las metas académicas planificadas (Díaz 
Barriga Arceo y Hernández Rojas, 2006; Monereo, 1990). Su empleo está asociado a la interacción con Procesos Cognitivos Básicos (atención, percepción, codificación, memoria, entre otros), conocimientos previos, estratégicos y metacognitivos; pero además, con la regulación de los factores motivacionales, afectivos y contextuales que tienen una gran influencia sobre el aprendizaje (Pintrich y De Groot, 1990; Pintrich, 2000).

Así como es importante que el sujeto que aprende se esfuerce por ser estratégico, también lo es la estimulación que puede efectuar el agente de enseñanza por medio de las acciones que lleve a cabo. Esto conduce a hacer referencia a las estrategias de ense$\tilde{n} a n z a$, que son los recursos y procedimientos que los enseñantes utilizan para regular sus acciones y las variables del contexto con el objeto de promover aprendizajes significativos en los estudiantes (Díaz Barriga Arceo y Hernández Rojas, 2006: 430).

Existen numerosas investigaciones y propuestas pedagógicas dirigidas a suscitar docentes más estratégicos, cuyo origen podría encontrarse en la inquietud que está latente en todo profesional que valore la docencia, y que analiza de qué modo mejorar su práctica para ayudar a que sus alumnos aprendan su materia y adquieran un juicio crítico; y también por ser una competencia requerida en la sociedad del conocimiento con el objeto de aprovechar cada situación de enseñanza-aprendizaje y de formar estudiantes más autónomos. De todas ellas, hemos seleccionado algunas ideas desarrolladas por Pintrich (2000) en su modelo del aprendizaje autorregulado, para luego aplicarlas a la situación del profesional docente.

Pintrich (2000) considera el aprendizaje autorregulado como la capacidad que tiene un sujeto para seleccionar metas académicas y regular los factores cognitivos, motivacionales, afectivos y contextuales para poder alcanzarlas. En el proceso regulatorio, cada uno de estos aspectos conforma cuatro áreas que son controladas a través de distintas fases, en una sucesión temporal de subprocesos con funciones específicas y complementarias (Pintrich, 2000; Schunk, 2005).

La primera fase, denominada de Previsión, planificación y activación, conlleva programar, establecer metas de aprendizaje, considerar cómo incide el conocimiento personal, el contexto y los conocimientos previos sobre las tareas a realizar.

Durante el ejercicio de la enseñanza, al activarse esta etapa, el docente debería planificar los objetivos que quiere alcanzar con el grupo de alumnos que tiene a cargo, considerar el contexto áulico en el que se desarrollará la clase, prever los recursos materiales y teóricos necesarios para dictar cada tema previsto en el programa, tener en cuenta los conocimientos previos de los estudiantes, entre otros aspectos.

En la segunda fase, de Monitoreo, se activa una mayor conciencia metacognitiva con la cual se realiza un seguimiento sobre los factores personales, la tarea y el contexto. Si esto se aplica al docente, éste debería ahondar sobre los conocimientos que tiene en relación a las estrategias de enseñanza que utiliza, sobre la finalidad con que las emplea, sobre cómo las selecciona y aplica en cada situación y contexto de enseñanza (Bernardo Carrasco, 2008).

La tercera fase, de Control, como su nombre lo indica, tiene la función de controlar y regular las cuatro áreas ya mencionadas que conforman el proceso. En la tarea docente, se concretaría en regular los efectos producidos por la modalidad con la que transmite los contenidos planificados y las estrategias seleccionadas.

Finalmente, en la cuarta fase, de Reflexión y reacción, se evalúa la tarea realizada, el desempeño y el contexto para modificar aquellos elementos que pueden ser menos 
favorables para los futuros aprendizajes. Aquí el docente evaluará cómo ha dictado la clase, la forma con la que se vinculó y comunicó con los estudiantes y cuál ha sido la participación de estos últimos. Mucho mejor será si esta tarea la realiza en conjunto con los discentes, solicitándoles que expresen su opinión sobre el desarrollo del espacio compartido y en qué medida se han cubierto las expectativas con las que iniciaron el proceso.

En la medida que el profesor se auto conozca y se ejercite en la regulación de los matices que afectan a su función profesional, podrá desempeñarse en forma más estratégica; tarea que no deja de ser ardua y exigente porque conlleva la apertura de espacios destinados a la reflexión y a la evaluación de la propia actividad; para lo cual muchas veces no se tiene tiempo, más aún considerando las particularidades que tiene la tarea del profesor universitario, que en la mayoría de los países latinoamericanos se debe repartir entre el tiempo dedicado a la enseñanza y a otras actividades profesionales.

\section{2. ¿QUÉ OCURRE EN EL AULA UNIVERSITARIA? OBSERVACIONES REALIZADAS EN EL AULA. DISEÑO Y RESULTADOS}

A partir del marco teórico precedente y con el objetivo de conocer cuáles son los recursos didácticos que utilizan los docentes, se observaron en forma sistemática cuatro materias del cuarto año de la carrera de medicina utilizando como guía una planilla de observación elaborada $a d-h o c^{1}$, conformada por 80 indicadores organizados en tres dimensiones: Motivación, Estrategias de Aprendizaje y Personalización del Aprendizaje. La primera está formada por 6 indicadores (metas extrínsecas, metas intrínsecas, valor de la tarea, control de la tarea, autoeficacia, ansiedad), la segunda por 9 (repetición, elaboración, organización, pensamiento crítico, autorregulación metacognitiva, manejo del tiempo y del ambiente, regulación del esfuerzo, aprendizaje en grupo, búsqueda de ayuda) y la última por un solo indicador (Personalización del Aprendizaje).

Excepto en este último caso, para la redacción de los demás indicadores se tomaron como referencia las sub-escalas del cuestionario MSLQ (Motivated Strategies Learning Questionnaire, para su sigla en inglés ${ }^{2}$ ) (Pintrich, Smith, García y Mc Keachie, 1991) con el que puede obtenerse información sobre las estrategias metacognitivas, cognitivas y motivacionales que influyen en el aprendizaje autorregulado.

$1 \quad$ Ejemplos de ítemes:

“01. Ofrece material de estudio que conlleve un desafío y que permita el desarrollo de nuevos aprendizajes por parte de los estudiantes".

"09. Estimula a sus alumnos a que tomen conciencia del valor que tiene el aprendizaje de los contenidos de su materia a fin de que puedan utilizarlos en otros espacios curriculares".

"17. Manifiesta su confianza en los estudiantes de que podrán entender todos los temas (hasta los más difíciles) que se dictarán durante el año".

"21. Cuando toma un examen no remarca las consecuencias negativas que tendrán las malas calificaciones".

“24. Favorece la consolidación de los aprendizajes por medio de Actividades de Fijación (repaso, formulación de preguntas, reglas mnemotécnicas, autoevaluación)".

“47. Suscita el seguimiento sobre el propio desempeño, mediante la reflexión y la auto-evaluación”.

2 Traducción realizada por Daura, F. T. (Escala de Motivación y Estrategias de Aprendizaje). 
A fin de ponderar su confiabilidad, se empleó el alpha de Cronbach, que alcanzó un coeficiente de 0,8643 . Si se eliminan las variables autoeficacia y ansiedad -que es la estrategia de mayor incidencia en el índice final-, el alpha aumenta a 0,9006.

Para enriquecer la información recogida por medio de esta planilla, se tomaron notas descriptivas de la interacción en el aula entre los docentes y los alumnos y entre éstos entre sí.

Durante las observaciones se buscó considerar qué acciones efectuadas por los profesores podrían facilitar en los alumnos el desarrollo de algunas de las variables que intervienen en el aprendizaje autorregulado.

A fin de calcular los porcentajes, se sumó el total de las puntuaciones ( 3 a 0 ) y se lo dividió por la suma del puntaje máximo posible, a la que se le restó la sumatoria de la puntuación total de los ítems valorados como sin elementos de juicio en las observaciones realizadas ${ }^{3}$.

A los porcentajes de las variables de cada dimensión se los representó en distintos gráficos que facilitan su lectura y que se interpretan a continuación.

Los resultados de la dimensión Motivacional, constituida por los 6 indicadores señalados, se representan en el primer gráfico en el que cada asignatura observada se identifica con un color distinto que se mantendrá en las sucesivas representaciones para facilitar su comprensión.

\section{Gráfico I}

Resultados comparativos de las observaciones. Dimensión Motivacional

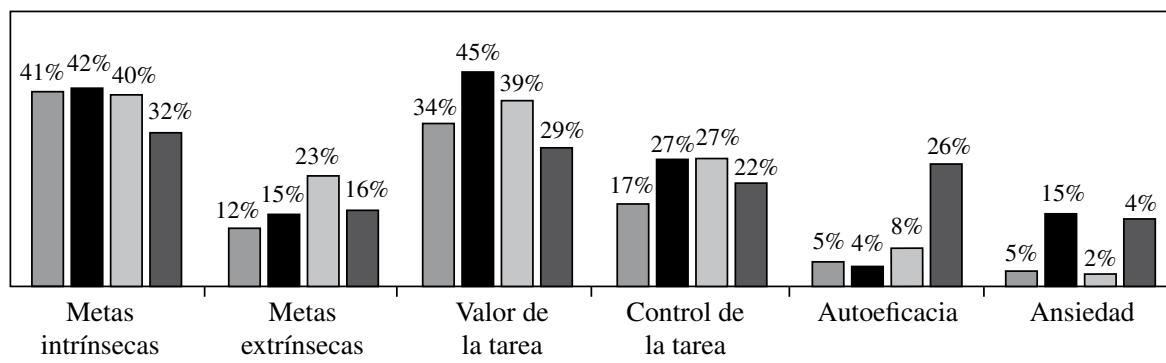

Motivación

\section{Medicina Interna II $\square$ Cuestiones de Bioética $\square$ Cirugía $\square$ Farmacología II}

Fuente: Elaboración propia a partir de los datos recolectados.

3 Por ejemplo, en la asignatura "Medicina Interna II, en la dimensión "metas extrínsecas" el docente obtuvo 14 puntos, siendo el puntaje máximo posible (4 indicadores por 16 observaciones, con una puntuación de 3 -Muy bueno-) de 192 puntos.

A raíz de que no se registraron datos en 26 ocasiones, al puntaje máximo posible se restaron 78 puntos (que se obtuvieron multiplicando las 26 ocasiones calificadas como "sin elementos de juicio" por 3, que es la puntuación máxima). De este modo, el puntaje máximo posible en esta materia es de 192, número considerado para determinar el porcentaje. 
Según la prueba de diferencia de proporciones, en cada una de las cuatro variables la desigualdad encontrada entre el porcentaje mayor y el menor no es estadísticamente significativa ${ }^{4}$.

En Metas intrínsecas, las tres primeras asignaturas aludidas tienen una puntuación semejante, lo que se debe a que los docentes presentaron materiales y temas que involucraban algún tipo de desafío y de dificultad para los estudiantes o a que dedicaban tiempo para explicar más profundamente alguno de los temas tratados.

En todas las asignaturas se observó un porcentaje menor en cuanto a la estimulación de metas extrínsecas ya que en contadas ocasiones se percibió que se orientara a los alumnos sobre la importancia de seleccionar metas de aprendizaje o que se tuviera en cuenta sus motivaciones personales, entre otros factores.

La valoración de la tarea fue estimulada en todos los espacios curriculares, sobre todo en Cuestiones de Bioética, en la cual se constató una gran preocupación por parte de los docentes de que los estudiantes se concientizaran sobre la importancia que tiene el desarrollo de una postura ética frente a la futura profesión y a situaciones concretas que deberán revolverse. Del mismo modo, todos los docentes demostraban un gran interés por el dictado de su materia y por el aprendizaje de los contenidos que forman parte de su programa de estudios.

La disminución de los porcentajes de la variable Creencias de control del aprendizaje se debe a que en pocas situaciones los docentes promovieron en los alumnos el estudio personal como un medio importante para su aprendizaje, esto tal vez por considerar que por encontrarse en el nivel universitario poseen una mayor autonomía y consecuentemente no necesitan de un apoyo en este sentido, cuestión que aquí se hipotetiza y para corroborarla podrían efectuarse entrevistas en profundidad. Por esta misma razón, tal vez en ningún momento responsabilizaron a los estudiantes sobre su progreso académico o sugirieron una mayor dedicación de tiempo para estudiar los temas dictados en cada clase, mediando la circunstancia de la proximidad de una fecha de examen parcial o final.

En Autoeficacia los porcentajes disminuyen aún más en las materias Medicina Interna I, Cuestiones de Bioética y Cirugía.

Las situaciones en las que los profesores expresaron su optimismo por el buen rendimiento que lograrían los estudiantes, o bien su confianza en que todo el curso entendería los temas a dictar, sólo se registraron en el inicio de algunas de las materias o en fechas cercanas a las fechas de exámenes.

Con respecto a la Ansiedad, la reducción de los porcentajes sería positiva ya que explicaría que por lo general, durante las clases y momentos de evaluación se generaría un clima sereno, tranquilo, distendido y de confianza, considerando que los estudiantes son capaces de obtener buenas calificaciones y que pueden comprender los contenidos de cada espacio curricular.

La dimensión Estrategias de aprendizajes, conformada por una mayor cantidad de indicadores (9 en total), se representa en los gráficos II y III.

4 En Metas Intrínsecas, entre Cuestiones de Bioética y Farmacología II, z = 0,058.

En Metas Extrínsecas, entre Cirugía y Medicina Interna II, z $=0.86$.

Valor de la Tarea, entre Cuestiones de Bioética y Farmacología II, Z = 0,9362.

Control de la Tarea, entre Farmacología y Medicina Interna II, $Z=0,3880$.

En Autoeficacia, entre Medicina Interna II y Farmacología II, $Z=1,88$.

Ansiedad, entre Cuestiones de Bioética y Medicina Interna II, Z = 0,044139. 
En el caso de estas variables, se halla una diferencia estadísticamente significativa $(Z=1,98)$ en Pensamiento Crítico entre Cuestiones de Bioética y Farmacología, a favor de la primer asignatura ${ }^{5}$.

\section{Gráfico II}

Resultados comparativos de las observaciones. Dimensión Estrategias de Aprendizaje - I

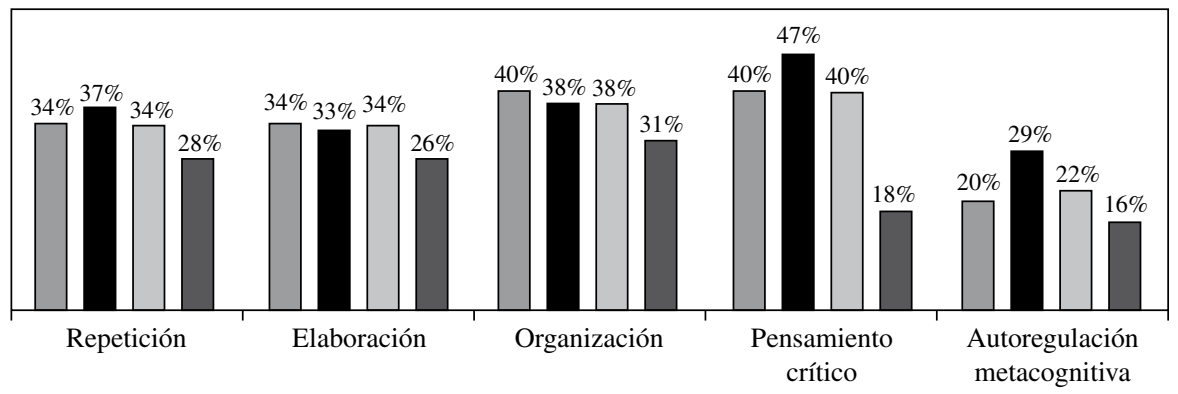

Medicina Interna II $\square$ Cuestiones de Bioética $\square$ Cirugía $\square$ Farmacología II

Fuente: Elaboración propia a partir de los datos recolectados.

El indicador Repetición se refiere a las oportunidades brindadas por los docentes para consolidar el aprendizaje de los contenidos estudiados durante las clases mediante actividades de fijación, orientaciones sobre cómo recordar conceptos y términos importantes (cuestión que es fundamental en Farmacología, por ejemplo).

Esta variable se hizo más notoria en la materia Cuestiones de Bioética, en oportunidad de presenciar unas clases previas a un examen parcial en las que se repasaron los temas dictados durante el cuatrimestre.

En las demás clases observadas, los docentes optaron por repasar muy sintéticamente los contenidos tratados en anteriores oportunidades.

En cuanto a la Elaboración de contenidos, esta estrategia fue más promovida en Medicina Interna II y en Cirugía, mediante indicaciones concretas sobre cómo estudiar reuniendo información de distintas fuentes bibliográficas, estableciéndose vinculaciones entre los contenidos estudiados en estos y otros espacios curriculares. En el caso particular

5 En Repetición, entre Cuestiones de Bioética y Farmacología II, Z = 0,54.

En Elaboración, entre Medicina Interna II y Farmacología II, Z = 0,54.

En Organización, entre Medicina Interna II y Farmacología II, $Z=0,17$.

En Autorregulación Metacognitiva, entre Cuestiones de Bioética y Farmacología II, Z = 0,87.

En Manejo del tiempo y ambiente de estudio, entre Cuestiones de Bioética y Medicina Interna II, Z = 0,27.

En Regulación del esfuerzo, entre Cuestiones de Bioética y Farmacología II, Z = 0,14.

En Trabajo en grupo, entre Cirugía y Cuestiones de Bioética, $\mathrm{Z}=0,85$.

En Búsqueda de ayuda, entre Cuestiones de Bioética y Medicina Interna II, Z =0,42. 
de la segunda materia mencionada, la elaboración se promovió mayormente por medio de consignas claras y concretas que los docentes planteaban para que los estudiantes integraran los conocimientos y los reformularan en forma personal, con criterio propio. Asimismo, en todas las clases se utilizaron esquemas, clasificaciones, cuadros y redes semánticas para organizar los conocimientos.

Otro punto a destacar es que ningún docente promovió la utilización de técnicas de estudio en los estudiantes, punto que posiblemente esté vinculado con la idea expresada unos párrafos más arriba sobre la creencia de que los alumnos no necesitarían una orientación al respecto por tener desarrollada una mayor autonomía.

La estrategia de Organización llega a tener una diferencia de 10 puntos por arriba de los demás indicadores, siendo de $40 \%$ en Medicina Interna y de porcentajes levemente inferiores en Cuestiones de Bioética y en Cirugía.

En estas asignaturas se explicitaron los objetivos a alcanzar en cada una; se advertía que las clases estaban planificadas y organizadas con una lógica y coherencia interna y se empleaban diversos recursos para facilitar la organización de los alumnos (por ejemplo, proporcionando el programa y el cronograma de la materia, utilizando mapas conceptuales o repasando los temas dictados entre una clase y otra).

En Farmacología II, la organización principalmente se efectuó en forma verbal, sintetizando los contenidos dictados entre cada sesión.

La estrategia de Pensamiento Crítico es el indicador que tiene porcentajes más altos en Medicina Interna I, Cuestiones de Bioética y en Cirugía, como consecuencia de la metodología adoptada en cada espacio curricular caracterizada por el predominio del método del caso, de la búsqueda del diálogo con los estudiantes, de la promoción de un juicio crítico y argumentativo. En Farmacología II, la disminución del porcentaje, no se debería a la ausencia de técnicas y procedimientos como los indicados, sino a su corta carga horaria y a la gran cantidad de contenidos de carácter predominantemente fáctico o informativo.

Aunque en comparación con los demás indicadores analizados tiene porcentajes menores, es en Cuestiones de Bioética en donde la Autorregulación Metacognitiva tiene la mayor valoración.

En ninguna de las clases se percibió que los docentes promovieran en los estudiantes conductas de seguimiento sobre su propio desempeño mediante la reflexión y la autoevaluación.

El indicador Manejo del Tiempo y el Ambiente de estudio es en el que tienen un menor porcentaje todas las materias con excepción de Cuestiones de Bioética. Concretamente se refiere a la orientación que puede brindarse a los estudiantes sobre factores témporoespaciales que afectan sobre el aprendizaje: lugar y tiempo apropiados para estudiar, importancia de planificar el estudio con el objeto de dejar espacio para otras actividades, organización de cada clase y del material de estudio sugerido, aprovechamiento del horario disponible, seguimiento de la asistencia de los alumnos, entre otros puntos.

La Regulación del Esfuerzo hace alusión al control de las energías invertidas para realizar actividades académicas y que en la interacción entre los docentes y los alumnos se podría concretar en estimular a estos últimos para que den prioridad al estudio y a las tareas solicitadas, o bien en el tiempo dedicado para preparar las clases y que se demuestra en la variación de los recursos (materiales y humanos) y en las técnicas desplegadas. 


\section{Gráfico III}

Resultados comparativos de las observaciones. Dimensión Estrategias de Aprendizaje - II

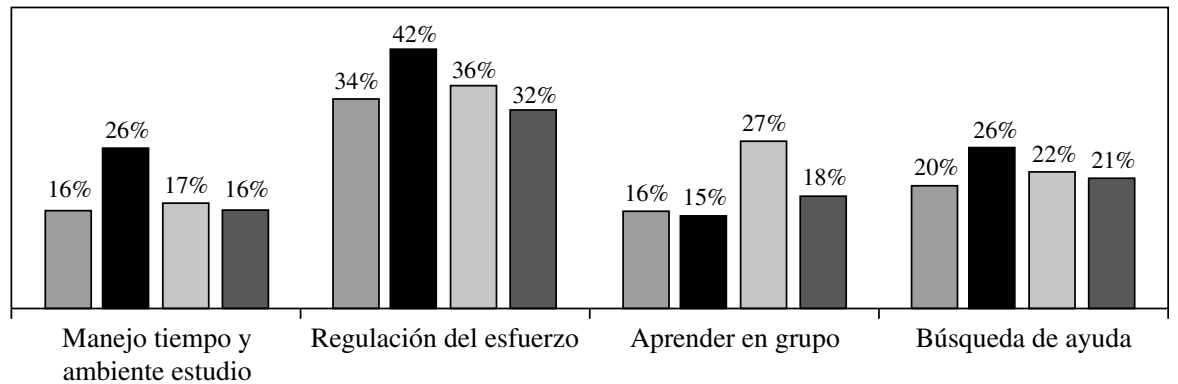

Medicina Interna II $\square$ Cuestiones de Bioética $\square$ Cirugía $\square$ Farmacología II

Fuente: Elaboración propia a partir de los datos recolectados.

En comparación con los demás indicadores, en éste todas las materias obtuvieron un alto porcentaje.

El Aprendizaje en Grupo se observa cuando se proponen actividades que estimulan la colaboración, el trabajo en equipo entre los alumnos y la discusión de contenidos de la materia u otros vinculados a ésta. Esta variable se favoreció en el espacio curricular de Cirugía, en el que los docentes solicitaron a los estudiantes que expusieran clases en pequeños grupos de trabajo, comentaran lo que habían aprendido durante las horas prácticas desarrolladas por equipos en los hospitales.

La propiciación de la Búsqueda de ayuda por parte de los docentes se realizó mayormente al iniciar o al terminar las horas de clase, momentos en los que preguntaban a los estudiantes si habían comprendido todos los temas dictados o en los que se mostraban disponibles para responder las consultas que se acercaban a hacerles.

La dimensión Personalización de los aprendizajes, cuyos resultados se muestran en el gráfico IV, se dirige a evaluar todas aquellas acciones que el docente realiza y los recursos didácticos que despliega para conocer más a los alumnos y para brindarles una educación integral ${ }^{6}$.

Tanto el conocimiento de cada uno o de la mayoría de los estudiantes, la facilitación de su participación activa en el aula, la organización flexible de la clase, la clara preocupación por la situación personal del grupo y de cada estudiante, la estimulación del trabajo que realizan para que desarrollen progresivamente su autonomía, como así también la propuesta de actividades significativas y que estimulen el desarrollo de los distintos aspectos de la persona (como el intelectual, espiritual, social, motivacional y

Se concibe que una educación integral es aquella que tiende al logro de la unificación de todos los aspectos de la vida del hombre a través del desarrollo de todas sus potencialidades. Es lo contrario a una suma de acciones y de contenidos educativos (García Hoz, 1988). 
Gráfico IV

Resultados comparativos de las observaciones. Dimensión Personalización del Aprendizaje

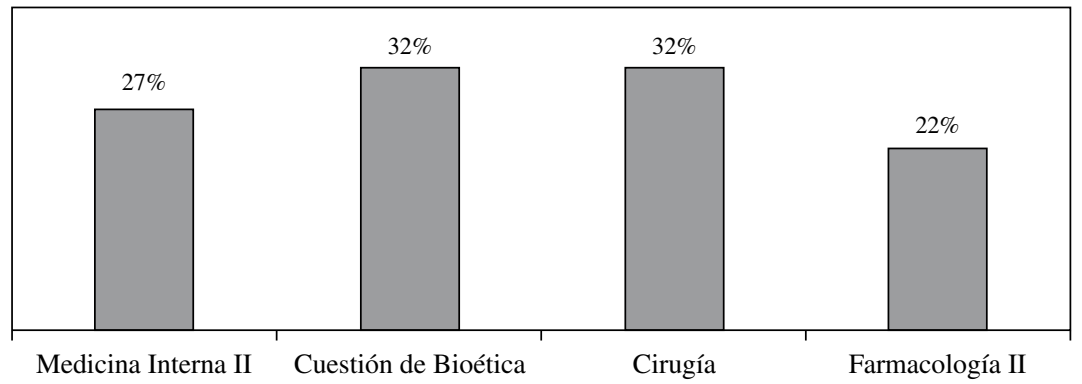

Fuente: Elaboración propia a partir de los datos recolectados.

afectivo) son formas a través de las cuales se promueve una mayor personalización del proceso de aprendizaje.

Por sus características, en donde más se ha visto favorecida esta dimensión fue en Cuestiones de Bioética y en Cirugía, asignaturas en las que explícitamente se desplegaron algunos de los puntos referidos.

A fin de establecer si estas diferencias son estadísticamente significativas, se aplicó la prueba de Mann-Whitney por dos niveles de personalización del aprendizaje -más alto y más bajo- (Cuestiones de Bioética y Cirugía en el primer grupo; Medicina Interna II y Farmacología II en el segundo) en todas las variables de la dimensión motivacional y de estrategias de aprendizaje.

En dos de estas variables se obtuvo una diferencia estadísticamente significativa: en pensamiento crítico $(U=26,50 ; p=0,001)$ y en valoración de la tarea $(U=52,50$; $\mathrm{p}=0,05)$.

Algunos de los factores que pudieron haber obstaculizado la personalización del aprendizaje son la corta duración de cada espacio curricular, la presencia de varios docentes con carácter de invitados y una gran carga de contenidos por dictar en cada materia.

La información recogida por medio de las observaciones efectuadas permite conocer en mayor medida los recursos didácticos que utilizan los docentes para favorecer la autonomía en los alumnos y el proceso de enseñanza-aprendizaje.

Los datos obtenidos en cada una de las variables que en ningún caso llega al 50 por ciento, hacen pensar en la posibilidad de implementar algún mecanismo de orientación docente que se dirija a brindar destrezas que favorezcan la autoobservación, el autoconocimiento y la regulación del propio desempeño profesional, de manera de hacerlo más estratégico y eficaz. Del mismo modo, puede ayudar a que los profesores tomen una mayor conciencia de que es en el mismo espacio áulico en el cual pueden orientar los aprendizajes que desarrollan los estudiantes, por medio de las actividades que proponen y del discurso verbal o no verbal que desplieguen. 


\section{CONCLUSIONES}

En el presente trabajo hemos hecho énfasis sobre la consideración de la didáctica como una disciplina pedagógica y de fuerte carácter aplicativo, que puede guiar la actividad del docente universitario, de forma de ayudarlo a ser verdaderamente estratégico a fin de estimular el aprendizaje autónomo en los estudiantes.

Con el objetivo de conocer los recursos que utilizan los docentes, se observaron en forma sistemática cuatro materias del cuarto año de la carrera de medicina, utilizando como guía una planilla de observación que se diseñó para el caso y que se organizó en tres dimensiones: Motivación, Estrategias de Aprendizaje y Personalización del Aprendizaje. Con excepción de los indicadores de esta última, para la redacción de los demás se tomaron como referencia los que forman parte del cuestionario MSLQ (Motivated Strategies Learning Questionnaire, para su sigla en inglés ${ }^{7}$ ) (Pintrich, Smith, García y Mc Keachie, 1991).

Para ponderar la confiabilidad del instrumento utilizado, se empleó el alpha de Cronbach, el cual alcanzó un coeficiente de 0,8643 , considerado por la teoría psicométrica como "considerable".

Luego de aplicar la prueba de diferencia de proporciones, no se encontraron diferencias estadísticamente significativas entre el porcentaje mayor y el menor obtenido entre las materias en las variables observadas en la dimensión motivacional (Metas Intrínsecas; Metas Extrínsecas; Valor de la Tarea; Control de la Tarea; Autoeficacia; Ansiedad).

Tampoco se hallaron diferencias estadísticamente significativas en los ítemes de la dimensión estrategias de aprendizaje (Repetición; Elaboración; Organización; Autorregulación Metacognitiva; Manejo del tiempo y ambiente de estudio; Regulación del esfuerzo; Trabajo en grupo; Búsqueda de ayuda); con excepción de la variable Pensamiento Crítico $(\mathrm{Z}=1,98)$.

La Personalización del Aprendizaje se vio favorecida en las materias "Cuestiones de Bioética" y "Cirugía", en las que explícitamente se desplegaron más estrategias dirigidas a favorecer esta dimensión. Entre otros aspectos, se considera que existen algunos factores que pueden haberse convertido en obstaculizadores de este aspecto, como la escasa duración de los espacios curriculares, la presencia de varios docentes invitados y una gran carga de contenidos por dictar en cada materia.

Los datos obtenidos en cada una de las variables han permitido conocer los recursos que los docentes utilizan para promover las variables mencionadas, para así llegar a efectuar orientaciones que hagan más estratégico su desempeño.

\section{REFERENCIAS BIBLIOGRÁFICAS}

García Hoz, V. (1988). Educación Personalizada (7ma ed.). Bogotá: Grupo Editor Quinto Centenario. Bernardo Carrasco, J. (Edit.) (2008). Cómo personalizar la educación. Una solución de futuro. (1 ${ }^{\text {era }}$ Edición) Ed. Narcea: Madrid.

Díaz Barriga, Á. (2009). Pensar la didáctica. Buenos Aires: Amorrortu editores.

$7 \quad$ Traducción realizada por Daura, F. T. (Escala de Motivación y Estrategias de Aprendizaje). 
Díaz-Barriga Arceo, F. y Hernández Rojas, G. (2006). Estrategias docentes para un aprendizaje significativo. Una interpretación constructivista. (2da edición). México: McGraw-Hill Interamericana Editores.

Medina Rivilla, A. y Salvador, F. (Coord.). (2002). Didáctica General (1era edición). Madrid: Pearson Educación.

Medina Rivilla, A. y Salvador Mata, F. (2005) (Coord.). Didáctica General. (Última reimpresión). Madrid: Prentice-Hall.

Monereo, C. (1990). Las estrategias de aprendizaje en la Educación formal: enseñar a pensar y sobre el pensar. Infancia y Aprendizaje. 50, 3-25.

Monereo, C., Badia, A., Bilbao, G., Cerrato, Ma. y Weise, C. (2009). Ser docente estratégico: cuando cambiar la estrategia no basta. Cultura y Educación, 21 (3), 1-20.

Pintrich, P. (2000). The role of goal orientation in self-regulated learning. En: Boekaerts, M., Pintrich, P.R. \& Zeidner, M. (Editors). Handbook of Self - Regulation. (451-502). San Diego: Academic Press.

Pintrich, P.R. y de Groot, E.V. (1990). Motivational and Self-Regulated Learning Components of Classroom Academic Performance. [Versión electrónica] Journal of Educational Psychology, Vol. 82, No 1, 33-40.

Pintrich, P., Smith, D., García, T. \& McKeachie, W. (1991). A manual for the use of the Motivated Strategies for Learning Questionnaire (MSLQ). Michigan: National Center for Research to Improve Postsecondry Teaching and Learning. University of Michigan.

Schunk, D.H. (2005). Self-Regulated Learning: The Educational Legacy of Paul R. Pintrich. Educational Psychologist, vol. 40, n. 2, 85-94.

Steiman, J. (2008). Más d idáctica (en la educación superior). Buenos Aires: Mino y Dávila Editores. 